\title{
Lentiviral expressing shRNAs inhibit the replication of Contagious Ecthyma virus by targeting DNA polymerase gene
}

Leila Asadi Samani

Shahrekord University

Behnaz Saffar ( $\nabla$ saffar_b@sci.sku.ac.ir)

Shahrekord University

Azam Mokhtari

Shahrekord University

Ehsan Arefian

University of Tehran

Research article

Keywords: ORFV, Lentiviral plasmid, DNA polymerase. RNAi, TCID50, Real-Time PCR

Posted Date: December 7th, 2019

DOI: https://doi.org/10.21203/rs.2.18213/v1

License: (a) (i) This work is licensed under a Creative Commons Attribution 4.0 International License.

Read Full License 


\section{Abstract}

Background: Contagious ecthyma or Orf is known as a zoonotic disease remains prevalently worldwide despite the application of some control strategies against it. RNAi particularly shRNA provides us with the chance to tackle this obstacle by an encouraging new approach. The current study indicates the design and experiment of third-generation lentivirus packaging systems delivering shRNAs to inhibit ORFV replication and infection.

Methods: Given the importance of DNA-pol gene in virus replication, in this study, three shRNAs against this gene were designed and cloned into lentiviral vectors to stabilize the expression of shRNAs. After producing lentivectors expressing ORFV-DNA- pol in HEK293T cells, the synthesized shRNAs were applied to downregulate viral replication and gene expression. The reduction in viral titer and RNA was evaluated by TCID50 test as well as real-time RT-PCR. The results were then analyzed in comparison with the control group.

Results: Designed shRNAs significantly reduced virus yield approximately $90 \%$ to $97 \%$ and $96.8 \%$ to $99.4 \%$, respectively compared to the control groups (cells infected with ORFV and infected with ORFV and scrambled vector) by TCID50 test. Real-time RT-PCR revealed a dramatic reduction in the expression of viral RNA approximately $99 \%$ compared to cells infected with ORFV and from $92.6 \%$ to $99 \%$, respectively compared to cells infected with ORFV and scrambled vector.

Conclusions: Therefore, it can be stated that RNAi is capable of being used as a potent therapeutically option against viruses like ORFV.

\section{Background}

Orf Virus, also known as sheep pox, ecthyma contagiosum, and contagious pustular dermatitis, is a parapoxvirus causing contagious ecthyma [1]. The virus effects skin and mucosal membrane of ruminants and may be a zoonotic pathogen [2,3]. Dolorous skin lesions may lead to anorexia or starvation. Lesions on the udder and foot can cause the offspring stop and transient lameness, respectively $[3,4]$.

It is obvious that none of the existing vaccines induce complete long-term immunity and therefore vaccinated animals can be re-infected again. There is no effective vaccine to prevent ORFV disease in humans [5]. RNAi treatments allow for a faster and more cost-effective development of drugs as the ability to target each gene in the target tissue. In addition, because RNAi can simultaneously target several genes in a drug, they also promise a great deal to cure complex a challenging diseases, especially cancer and viral infections [6].

shRNA is an artificial RNA molecules with a hairpin loop that is transcribed by RNA polymerase in nucleus [6]. siRNA has been proven to have some limitations. First, it is a relationship between the concentration of siRNA and off-target effects. second, in cell division, the concentration of siRNA starts to decrease. 
Thus, stable cell line generation with the aim of continuous and long-term target gene knockdown is not possible [7]. The comparison of the efficiency of siRNA versus shRNA is difficult. But when both siRNA and shRNA are produced the same way, shRNA is described to be somewhat more effective. Compared to siRNA, shRNA is continuously synthesized in host cells, leading to longer gene silencing. The most important advantage of using shRNA is that it can be done on a viral vector and entered into many cell lines [6]. Applying viral vectors can potentially reduce the difficulties of transfecting cell lines, and it is beneficial for in vivo studies [8].

Lentiviruses are a subset of retrovirus that are capable of integrating into the genomes of both dividing and nondividing cells in order to earn a stable and long-term expression of shRNAs [9]. Other benefits of lentiviruses include the following: broad tropism, integration into the host genome providing durable expression of the encoded therapeutic protein, relatively easy production and the availability of large libraries of constructs[10].

In the present study, having designed shRNAs against ORF025 gene that encodes the DNA Pol in order to inhibit ORFV multiplication, we generated lentivirus expressing shRNAs in HEK 293 T cells. Finally, we evaluated the rate of ORFV replication in MDBK cells.

\section{Results}

\section{Reducing ORFV replication by lentiviral expressing shRNA}

The inhibition of ORFV replication using shRNAs was determined by EGFP expression in transduced cells with lentiviral vector and observed for CPE induced by ORFV. The results showed that cells infected with anti-ORFV shRNAs visibly reduced CPE $72 \mathrm{~h}$ after the infection, compared to cells of control group (MDBK cells infected with ORFV) (Fig. 1a). The real-time RT-PCR was used for estimating the effect of RNAi on ORFV replication and titration of virus was done $72 \mathrm{~h}$ after the virus challenge.

As showed in Fig. 1b, shRNAs 1, 2 and 3 markedly reduced the expression of viral RNA compared to control group (approximately $99 \%, 99 \%$ and $99 \%$, respectively compared to cells infected with ORFV and approximately $99 \%, 92.6 \%$ and $99 \%$, respectively compared to cells infected with ORFV and scrambled vector) $(P<0.05)$. In the TCID50 test, the shRNAs 1, 2 and 3 also significantly reduced virus yield compared to the control groups (approximately $96 \%, 97 \%$ and $90 \%$, respectively compared to cells infected with ORFV and approximately $99 \%, 99.4 \%$ and $96.8 \%$, respectively compared to cells infected with ORFV and scrambled vector) (Fig. 1c).

The results of CPE indicated a reduction in virus yields in each group.

\section{Reduction of ORFV DnaQ_like expression by lentiviral expressing shRNA}


After the infection of stably expressing ORFV DnaQ_like MDBK cells and controls (MDBK cells infected with ORFV and those infected with mock vector) by lentiviral mediated shRNAs, the expression of selected ORFV sequence was detected using real-time RT-PCR test.

As showed in Fig. 2, shRNAs 1, 2 and 3 significantly reduced the level of viral gene expression. The shRNAs 1, 2 and 3 markedly reduced the expression of the selected gene compared to the control groups (approximately $86.5 \%, 99 \%$ and $96.5 \%$, respectively compared to cells without any infection and approximately $99.9 \%, 92.6 \%$ and $99.9 \%$, respectively compared to cells infected with scrambled vector). $(P<0.05)$.

$<$ figure 1 near hear>

$<$ figure 2 near hear>

\section{Discussion}

Since ORFV is a zoonotic and highly contagious agent with a global spread, its host range is expanding, causing a significant economic impact on the livestock industry. The application of the control measures such as vaccination has not been successful to reduce its prevalence. As a result, finding a way for its treatment or control is of interest to scientists. In Iran, unfortunately, the disease is prevalent in both traditional and industrial animal husbandry. Although some traditional therapeutic measures are used, the treatment of acute ORFV infections is usually not effective $[11,12]$.

Recently, RNAi-based treatments against viral infections such as diseases caused by the members of Poxviridae have been reported [13]. Alkhalil et al. (2009) exerted siRNAs against 12 viral genes of Monkeypox virus and showed RNAi has a potential therapeutic strategy for Monkeypox and other orthopox viruses [14]. Dave et al. (2006) utilized siRNAs against E3L gene of Vaccinia virus. By using two human cell types, namely HeLa and 293T, their investigation indicated the role of E3L-specific siRNAs as a powerful antiviral drug for small pox and related pox viruses[15]. Zhao et al. (2012) put four shRNAs against a region of ORF095 gene encoding virion core protein and illustrated the widespread effect of DNA-based siRNA for inhibiting the replication of Goatpox virus [16]. In the case of ORFV, only one study was conducted by means of RNAi strategy. Wang et al, (2014) applied siRNAs against DNA pol gene of ORFV by using pMD-18T vector, which indicated approximately $73-89 \%$ less viral DNA in cells transfected with the siRNAs [32]. However, many investigations have been done to eradicate this contagious disease which have mostly been supportive. In this study, due to the lack of specificity and side effects of chemical drugs, an RNAi-based approach was used to suppress ORFV replication. In fact, the present study is the first survey to investigate the inhibitory influence of shRNA on ORFV replication thanks to the constant expression of the shRNAs that was obtained through the use of lentivectors. Since the third generation of lentivirus contains only $10 \%$ of the viral genome sequence, it provides more safety for therapeutic applications [17]. 
In one hand, appropriate target transcripts should be selected when it comes to RNAi-based therapeutics. In the other hand, in the case of viruses, conserved sequences of genes in various virus strains need to be targeted. Hence, in current study, the highest conserved sequences with serious functions in replication were selected.

Because of the superiority of the shRNA to siRNA in various aspects like more durability and less dose and fewer Off-target effects [18],in this study, we constructed shRNA structures against ORF025-DNA pol gene that could effectively down-regulate virus replication. As time passes, lentiviral vectors evolve as a beneficial tool to constant gene transfer into desired cells with a plentiful replicative potential. Indeed, due to their partly flexible genome, the capability of transducing both nondividing and dividing cells as well as their potential for cell-specific pseudotyping, lentivectors supply a precious resource for a wide variety of applications in experimental platforms and therapeutic settings [19]. In the present study, we applied pCDH-CMV-MCS-EF1-cGFP-T2A-Puro vector as a third-generation lentivector aiming to gain a stable expression of designed shRNAs in order to prevent ORFV replication for a long time.

Except a report for the preparation of an ORFV-based viral vector, we did not find any study in which ORFV genes were expressed using LVs. In 2003, Fischer et al. cloned ORFVVEGF-E and D-and Cglycoproteins of pseudorabies in separate lentiviral plasmids, and subsequently co-transfected them into Vero cells. Therefore, a new viral vector based on Parapaxviruses was created [20].

Several cell lines expressing sub-replicons of some viruses have been developed to evaluate therapeutic and prophylactic therapies against viruses. For example, Basagoiti et al. (2009) cloned the target gene segments of Western Neil's virus into appropriate plasmids and produced a cell line expressing the viral sub-replicons after transfection. This cell line was successfully applied to evaluate the chemical inhibitors of the WNV epidemic strains [21].Kumar et al. (2006) found that lentivector pseudotypes that use RV-G instead of VSV-G are capable of delivering a specific RNAi induction in neuronal cells and significantly decrease the vector dose needed to reduce the target gene [22].Wang et al. (2013) produced U6 shRNA induced by lentivectors expressing GFP as a reporter protein. After cloning the nucleoprotein and matrix genes of Newcastle virus in the transfer plasmid and transfecting the appropriate cell line for expressing these genes, the shRNA molecules successfully inhibited the specific expression of these two genes and Newcastle Virus replication (90\% inhibition of proliferation) [23].

Prior to the validation of shRNAs against ORFV, an ORFV sub genomic expressing a cell line that provided an easy gene knockdown readout was used to help identify potentially active molecules [24]. The data presented here showed that using this method is useful for screening functional shRNAs. Lantermann et al. (2007) found that the expression of early and late genes of vaccina virus could be inhibited by siRNA. They used an evaluation method by producing the cell line expressing vaccinia sub genomic replicons for the specific evaluation of siRNA [25].Kilcher et al. (2014) applied RNAi to detect AAA + ATPase D5 as an uncoating factor of vaccinia virus. In this study, the specific evaluation of the interfering molecules was performed using a sub genomic replicon expressing cells [26]. 


\section{Conclusion}

In conclusion, the design of shRNA expressed by lentiviral vector illustrated the down - regulation by realtime RT-PCR and viral load methods. Overall, ORFV-ShRNA2 had a better suppressive effect than two other anti ORFV-ShRNAs.

\section{Methods}

\section{Design and Preparation of shRNA Molecules}

In this study, first of all, the shRNAs targeting sequences ORF025-DNA po/ gene were designed by means of three online design algorithm, namely: BLOCK-iTRNAi Designer (http://rnaide signer.lifetechnologies.com/rnaiexpress/),WIsiRNA Selection Program (http://sirna.wi.mit.edu/home.php) and siRNA Wizard Software (www.invivogen.com/sirna-wizard). Having more precisely investigated the sequence and structural shRNA design rules, we selected the most potent ones [7,27-29]. Apart from considering shRNA design tools, the proposed shRNAs were manually surveyed according to the parameters suggested by TomTuschl's rules [27], Mcintyre et al. [28] and Taxman et al. [7].

A BLAST search needs to be performed in order to eliminate shRNAs having much homology with sheep genomic and transcripts database (http://blast.ncbi.nlm.nih.gov/), and fortunately no homologies were observed.

\section{Production of lentivector expressing shRNAs and ORFV-DNA polymerase}

Three shRNA molecules were designed based on the ORFV-OV-SA00- DNA pol gene (accession number AY386264.1). Synthesized shRNAs were cloned separately into plasmids PCDH-CMV-MCS-EF1-cGFP-T2APuro to the downstream of the CMV promoter (which was kindly provided from Bonbiotech company). The map of the vector is illustrated in figure3. Furthermore, DnaQ_like exonuclease domain of ORFV-DNA pol gene was synthesized and cloned in the same lentivector. The lentivector used in this study, contained the CopGFP gene under the control of an EF1 promoter in order to pursue shRNA transfection efficiency. It is worth mentioning that the lentivector contained the gene of puromycin resistance cassette as well. All chemically-synthesized oligonucleotides were ligated into the lentivector which had been digested by $E C O R I$ and $B a m H$. Afterwards, lentivectors ligated in the previous step were transformed into the Escherichia coli strain DH5a. After verifying the accuracy of cloning by sequencing, the accurate clones were used for transfection. As a mock (no shRNA GFP lentivector), we used pEZX-MR03 that contained the EGFP gene under the control of a CMV promoter lacking any significant shRNA sequences [30].

$<$ figure 3 near here $>$

\section{Cells and virus propagation}

MDBK cells (NBL-1; ATCC Number: CCL-22) were cultured in DMEM (Gibco, America, Catalog No. 11612800) with $10 \%$ FBS (Gibco, America, Catalog No. 106- 10270) 2 mM L-glutamine, 1X penicillin 
streptomycin (Sigma, America Catalog No. 116-12800) as well as $2.5 \mathrm{mg} / \mathrm{L}$ amphotericin B at $37^{\circ} \mathrm{C}$ with $5 \% \mathrm{CO} 2$ incubator [31]. ORFV-SA00 standard strain was kindly provided by Dr. Asgari (Agricultural Research Service, United States Department of Agriculture, Greenport, New York). The titer of the virus stock was $8.91 \times 10^{4} \mathrm{TCID} 50 / \mathrm{ml}$. ORFV-SA00 was used at $100 \mathrm{TCID} 50$ for in vitro infection experiments [32].

\section{Packaging of lentiviruses}

In the present study, third-generation lentivirus packaging systems was applied in order to generate designed shRNAs and ORFV DnaQ_like expressing lentiviral vectors.

Having cultured HEK 293 T cells (ATCC Number: CRL-1573), we co-transfected the cells with a mixture of three vectors namely: $21 \mu \mathrm{g}$ psPAX packaging vector, $10.5 \mu \mathrm{g}$ pMD2.G encoding the vesicular stomatitis virus glycoprotein ( $V S V-G$ ) envelope and $21 \mu \mathrm{g} \mathrm{pCDH}$ (or pEZX-MR03 as a mock) transfer vector by means of Ca-Po4 reagent according to the bonbiotech (Iran) company's guidelines. The co-transfection was performed in 10-cm plates similar to the conditions mentioned in manufacturer's instructions in order to gain a confluency of approximately $70-80 \%$.

48 and $72 \mathrm{~h}$ after transfection, the GFP expression in transfected cells was monitored and photographed by a fluorescent microscope (Fig.4a). On the other hand, at the same time, the supernatant from these plates were collected and cleared by centrifugation $(1000 \mathrm{~g}, 15 \mathrm{~min})$ and were kept in $-70^{\circ} \mathrm{C}$ until the infection of MDBK cells [33].

\section{Preparation of MDBK cells expressing ORFV DnaQ_like and its challenge with shRNA}

To prepare MDBK cells expressing ORFV DnaQ_like, an amount of $10^{5}$ trypsinized MDBK cells were infected in a 6-well plate by lentiviral vectors $(\mathrm{MOI}=0.8)$, diluted in $1 \mathrm{~mL}$ of DMEM medium supplemented by $10 \%$ FBS. The medium was changed with $2 \mathrm{ml}$ of DMEM after $12 \mathrm{~h}$ of infection. At $48 \mathrm{~h}$ and $72 \mathrm{~h}$ after infection, MDBK cells were observed using a fluorescent microscope [34]. Infected cells were identified using RT-PCR. For RT-PCR, RNA was isolated from infected cells and then underwent a DNase treatment. Then, RT- PCR was carried out using the following primers: Forward: 5'- GGGACCGAGACAGTCAACTT -3' and Reverse: $5^{\prime}$ - GGTCCCGTTGTTGTTGTTGA $-3^{\prime}$. The PCR thermal cycle programs include denaturation at $95^{\circ} \mathrm{C}$ for $2 \mathrm{~min}$ followed by 30 cycles at $95^{\circ} \mathrm{C}$ for $30 \mathrm{~s}, 52^{\circ} \mathrm{C}$ for $30 \mathrm{~s}$, and $72^{\circ} \mathrm{C}$ for $30 \mathrm{~s}$, followed by a final extension at $72^{\circ} \mathrm{C}$ for $5 \mathrm{~min}$. The positive (MDBK infected with ORFV) and negative (MDBK cells without any infection) controls were used in each test.

To infect MDBK cells expressing ORFV DnaQ_likeby lentiviruses, the cells ( $90 \%$ confluent) were infected in a 6-well plate by lentivira/ vectors $(\mathrm{MOI}=0.8$ ), diluted in $1 \mathrm{ml}$ of DMEM medium supplemented by $3 \% \mathrm{FBS}$. The medium was changed with DMEM supplemented by $10 \%$ FBS and $1 \mathrm{X}$ penicillin streptomycin after 12 $\mathrm{h}$ of infection. The cells were re-infected after $12 \mathrm{~h}$ post changing medium with the same method and MDBK cells were maintained in DMEM, $10 \%$ FBS containing $1 \mathrm{X}$ penicillin streptomycin (Sigma). For each shRNA, three wells were considered [35]. 


\section{Infection /challenge of lentivirus expressing shRNAs with ORFV}

$3 \star 10^{5}$ MDBK cells per 6 -well plates were seeded and cultured with the conditions mentioned in the previous part. The next day, after removing the culture medium, lentiviruses were inoculated into wells. For each shRNA, three wells were considered. To achieve more infecting efficiency, $24 \mathrm{~h}$ after the first infection with lentivirus, the infection repeated like that of the previous step.

$48 \mathrm{~h}$ after the first infection, MDBK cells were monitored under a fluorescent microscope to evaluate GFP expression (Fig. 4b). In case of observing the appropriate GFP expression, the challenge with ORFV was performed. After $72 \mathrm{~h}$, cells were monitored in terms of phenotypes and the development of CPE .MDBK cells infected with ORFV were considered as positive controls and MDBK cell infected with mock vector or without any viral infection were considered as negative controls [35].

$<$ figure 4 near hear>

\section{TCID50 Assay}

To determine the changes in CPE as well as the effects and reduction of ORFV titer,TCID50 test was performed according to the protocol after challenging with lentiviruses expressing shRNAs, [36]. TCID50 was performed in 96-well plates with three replicates per dilution and in triplicate for each infection condition. Finally, viral titers were calculated based on the Spearman-Karber formula [37].

\section{RT-qPCR}

Total RNA was extracted from MDBK cells using the RNeasy mini kit (Qiagen, Crawley, UK), 72h after the infection. The on-column DNase digestion (Qiagen, Crawley, UK) was used to remove contamination off DNA. The $c D N A$ was synthesized from $1 \mu \mathrm{g}$ total RNA by reverse transcriptase enzyme.

Reverse-transcription performed by Superscript II (Invitrogen) and oligo(dT) for $1 \mathrm{~h}$ at $42{ }^{\circ} \mathrm{C}$. RT-qPCR was performed using Power SYBR Green Mastermix (Applied Biosystems, Warrington, UK). The primers were designed using the GenScript real-time RT-PCR Primer Design web tool. The primer sequences were screened using a BLAST search to confirm its specificity and the PCR products were run on an agarose gel to confirm that products of the expected size were detected. The sequences of forward and reverse primers were as follows: Forward: 5'- GGGACCGAGACAGTCAACTT- 3', Reverse: 5'-

GGTCCCGTTGTTGTTGTTGA- 3', Bos Taurus GAPDH Forward: 5' - TGAGGACCAGGTTGTCTCCT- 3'and Bos Taurus GAPDH Reverse: 5'- CACCCTGTTGCTGTAGCCAAAT- 3'. The reactions were analyzed upon an iQ5 real-time RT-PCR detection system. The PCR reaction was started with 30 s minute denaturation at 95 ${ }^{\circ} \mathrm{C}$. 40 cycles of $95^{\circ} \mathrm{C}$ for $30 \mathrm{~s}, 52^{\circ} \mathrm{C}$ for $45 \mathrm{~s}$ and $72^{\circ} \mathrm{C}$ for $1 \mathrm{~min}$ were carried out. Cycle threshold (Ct) values were normalized to GAPDH, and a relative ORFV RNA level was done by the $\Delta \Delta \mathrm{Ct}$ method [38].The fold-change values relative to control were multiplied by 100 to obtain the reduction of percentage in ORFV replication. Experiments were performed in triplicate. The amplification was identified by melting curve profile. 


\section{Abbreviations}

ORFV: Contagious ecthyma virus; RNAi:RNA interference; shRNA:short hairpin RNA; siRNA:small interfering RNA; DNA Pol:DNA polymerase; HEK 293 T:Human embryonic kidney 293T; MDBK:MadinDarby Bovine Kidney; BLAST:Basic Local Alignment Search Tool; ORFV-SA00:standard strain of ORFV; $\mathrm{CMV}$ :Cytomegalovirus; CopGFP:green fluorescent protein cloned from copepod Pontellina plumata; EF1:elongation factor-1; EcoRl:a restriction endonuclease enzyme isolated from species E. coli; BamHI:restriction endonuclease enzyme isolated from species Bacillus amyloliquefaciens; DH5a:E.coli cell engineered to maximize transformation efficiency; GFP:green fluorescent protein; EGFP:enhanced GFP; DMEM:Dulbecco's Modified Eagle's medium; FBS:fetal bovine serum; TCID50:50\% Tissue culture Infective Dose; Ca-Po4:Calcium orthophosphates; MOI:multiplicity of infection; RT-PCR:Reverse transcription polymerase chain reaction; DNase:deoxyribonuclease; CPE:cytopathic effects; RTqPCR:Quantitative reverse transcription PCR; GAPDH:glyceraldehyde-3-phosphate dehydrogenase; E3L:Protein E3 gene of Vaccinia virus; HeLa:Henrietta Lacks' 'Immortal' Cells; WNV:Western Neil's virus; U6:a type III RNA polymerase III promoter;

\section{Declarations}

\section{Ethics approval and consent to participate}

Not applicable.

\section{Consent for publication}

Not applicable.

\section{Availability of data and materials}

The data and materials used and/or analyzed during the current study are available from the corresponding author on reasonable request.

\section{Competing interests}

The authors declare that they have no competing interests.

\section{Funding}

This study was funded by The Iranian National Science Foundation (INSF) (project number: 95849043) and Shahrekord University (grant number: 95GRN1M730).

\section{Authors' contributions}

All authors conceived and designed the experiments; LA, BS, AM, and EA performed the experiments; LA, $B S$, and AM wrote the paper. All authors read and approved the final manuscript. 


\section{Acknowledgment}

The authors would like to thank Shokoohi.Ehsan for grammatically revising the paper

\section{Authors' information}

${ }^{1}$ Department of Genetics, Faculty of Science, Shahrekord University, Shahrekord- Iran. ${ }^{2}$ Biotechnology Research Institute, Shahrekord University, Shahrekord, Iran

\section{References}

1. Biazar TJE: Erythema multiforme as a result of Orf disease; a case report. Emergency. 2016; 4(3):163.

2. Kumar R, Trivedi R, Bhatt P, Khan S, Khurana S, Tiwari R, Karthik K, Malik Y, Dhama K, Chandra RJAAVS: Contagious pustular dermatitis (orf disease)-epidemiology, diagnosis, control and public health concerns. Adv Anim Vet Sci. 2015; 3(4):560-569.

3. S. Nandi UKD, Sumit Chowdhury: Current status of contagious ecthyma or orf disease in goat and sheep-A global perspective. Small Ruminant Research. 2011; 96(2-3):p. 73-82.

4. Mahmoud M, Abdelrahman K, Soliman HJRivs: Molecular and virological studies on contagious pustular dermatitis isolates from Egyptian sheep and goats. Research in veterinary science. 2010; 89(2):290-294.

5. Hosamani M, Scagliarini A, Bhanuprakash V, Mclnnes CJ, Singh RKJEroa-it: Orf: an update on current research and future perspectives. Expert review of anti-infective therapy. 2009; 7(7):879-893.

6. Haussecker DJJoCR: Current issues of RNAi therapeutics delivery and development. Journal of controlled release. 2014; 195:49-54.

7. Taxman DJ, Moore CB, Guthrie EH, Huang MT-H: Short hairpin RNA (shRNA): design, delivery, and assessment of gene knockdown. In: RNA therapeutics. Springer. 2010; 139-156.

8. Lambeth LS, Smith CA: Short hairpin RNA-mediated gene silencing. In: siRNA Design. Springer. 2013; 205-232.

9. Kong QJTJoci: RNAi: a novel strategy for the treatment of prion diseases. The Journal of clinical investigation . 2006; 116(12):3101-3103.

10. Seidlits SK, Gower RM, Shepard JA, Shea LDJEoodd: Hydrogels for lentiviral gene delivery. Expert opinion on drug delivery. 2013; 10(4):499-509.

11. Chi X, Zeng X, Li W, Hao W, Li M, Huang X, Huang Y, Rock DL, Luo S, Wang SJFim: Genome analysis of orf virus isolates from goats in the Fujian Province of southern China. Frontiers in microbiology. $2015 ; 6: 1135$.

12. Said AA, Mohamed SI, Elhamid NKA, Hosny WA, Baheeg EMJRiz: Trials for isolation of contagious pustular dermatitis virus (CPDV) from sheep in Ismailia governorate. Research in zoology. 2013; 3(1):10-14. 
13. Fischer LT, James QYJCr: RNAi, a new therapeutic strategy against viral infection. Cell research. 2004; 14(6):460.

14. Alkhalil A, Strand S, Mucker E, Huggins JW, Jahrling PB, Ibrahim SMJVj: Inhibition of Monkeypox virus replication by RNA interference. Virology journal. 2009; 6(1):188.

15. Dave RS, McGettigan JP, Qureshi T, Schnell MJ, Nunnari G, Pomerantz RJJV: siRNA targeting vaccinia virus double-stranded RNA binding protein [E3L] exerts potent antiviral effects. Virology. 2006; 348(2):489-497.

16. Zhao Z, Wu G, Zhu X, Yan X, Dou Y, Li J, Zhu H, Zhang Q, Cai XJVj: RNA interference targeting virion core protein ORF095 inhibits Goatpox virus replication in Vero cells. Virology journal. 2012; 9(1):48.

17. Segura MM, Mangion M, Gaillet B, Garnier AJEoobt: New developments in lentiviral vector design, production and purification. Expert opinion on biological therapy. 2013; 13(7):987-1011.

18. Mansoori B, Shotorbani SS, Baradaran BJApb: RNA interference and its role in cancer therapy. Advanced pharmaceutical bulletin. 2014; 4(4):313.

19. Schambach A, Zychlinski D, Ehrnstroem B, Baum CJHgt: Biosafety features of lentiviral vectors. Human gene therapy. 2013; 24(2):132-142.

20. Fischer T, Planz O, Stitz L, Rziha H-JJJov: Novel recombinant parapoxvirus vectors induce protective humoral and cellular immunity against lethal herpesvirus challenge infection in mice. Journal of virology. 2003; 77(17):9312-9323.

21. Puig-Basagoiti F, Qing M, Dong H, Zhang B, Zou G, Yuan Z, Shi P-YJAr: Identification and characterization of inhibitors of West Nile virus. Antiviral research. 2009; 83(1):71-79.

22. Kumar P, Lee SK, Shankar P, Manjunath NJPm: A single siRNA suppresses fatal encephalitis induced by two different flaviviruses. PLoS medicine. 2006; 3(4):e96.

23. Wang Z, Ding Z, Ding C, Yu S, Dang Y, Guo Y, Yang J, Meng Q, Liu J, Cong YJAd: Inhibition of Newcastle disease virus replication by lentivirus-mediated RNA interference. Avian diseases. 2013; 57(2):260-265.

24. Henry SD, Van Der Wegen P, Metselaar HJ, Tilanus HW, Scholte BJ, Van Der Laan LJJMT: Simultaneous targeting of HCV replication and viral binding with a single lentiviral vector containing multiple RNA interference expression cassettes. 2006; 14(4):485-493.

25. Lantermann M, Schwantes A, Sliva K, Sutter G, Schnierle BSJVr: Vaccinia virus double-stranded RNAbinding protein E3 does not interfere with siRNA-mediated gene silencing in mammalian cells. Virus research. 2007; 126(1-2):1-8.

26. Kilcher S, Schmidt FI, Schneider C, Kopf M, Helenius A, Mercer JJCh, microbe: siRNA screen of early poxvirus genes identifies the AAA+ ATPase D5 as the virus genome-uncoating factor. Cell host \& microbe. 2014;15(1):103-112.

27. www.protocol-online.org/prot/Protocols/Rules-of-siRNA-design-for-RNA-interference-RNAi-3210.html,2004/ TsRosdfRiRI-UoOAfU. 
28. Mcintyre GJ, Yu Y-H, Lomas M, Fanning GCJBmb: The effects of stem length and core placement on shRNA activity. BMC molecular biology. 2011; 12(1):34.

29. Baghban-Kohnehrouz B, Nayeri SJMbrc: Design, simplified cloning, and in-silico analysis of multisite small interfering RNA-targeting cassettes. Molecular biology research communications. 2016; 5(1):31.

30. Malekshahi SS, Salimi V, Arefian E, Fatemi-nasab G, Adjaminejad-Fard S, Yavarian J, Mokhtari-Azad TJMb: Inhibition of respiratory syncytial virus replication by simultaneous targeting of mRNA and genomic RNA using dual-targeting siRNAs. Molecular biotechnology. 2016; 58(11):767-775.

31. Zhao K, Li J, He W, Song D, Zhang X, Zhang D, Zhou Y, Gao FJVj: Cyclophilin B facilitates the replication of Orf virus. Virology journal. 2017; 14(1):114.

32. Wang G, He W, Song D, Li J, Bao Y, Lu R, Bi J, Zhao K, Gao FJAov: In vitro RNA interference targeting the DNA polymerase gene inhibits orf virus replication in primary ovine fetal turbinate cells. Archives of virology. 2014; 159(5):915-920.

33. Cockrell AS, Kafri TJMb: Gene delivery by lentivirus vectors. Molecular biotechnology. 2007; 36(3):184-204.

34. Barde I, Salmon P, Trono DJCpin: Production and titration of lentiviral vectors. Current protocols in neuroscience. 2010; 53(1):4.21. 21-24.21. 23.

35. Sastry L, Johnson T, Hobson M, Smucker B, Cornetta KJGt: Titering lentiviral vectors: comparison of DNA, RNA and marker expression methods. Gene therapy. 2002; 9(17):1155.

36. Kumar PJMM: Methods for rapid virus identification and quantification. 2013; 3:207.

37. Ramakrishnan MAJWjov: Determination of $50 \%$ endpoint titer using a simple formula. World journal of virology. 2016; 5(2):85.

38. Livak KJ, Schmittgen TDJm: Analysis of relative gene expression data using real-time quantitative PCR and the 2- $\triangle \triangle C T$ method. 2001; 25(4):402-408.

\section{Figures}


A

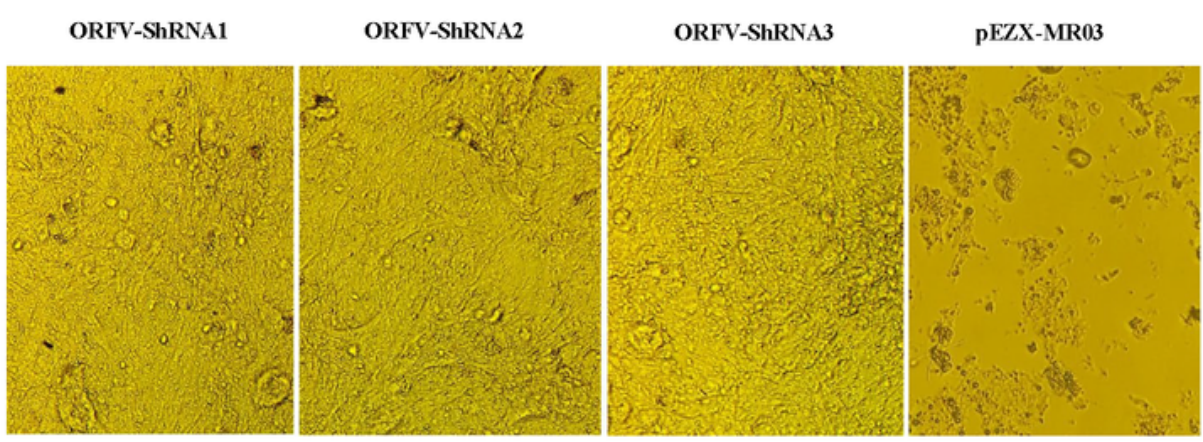

B
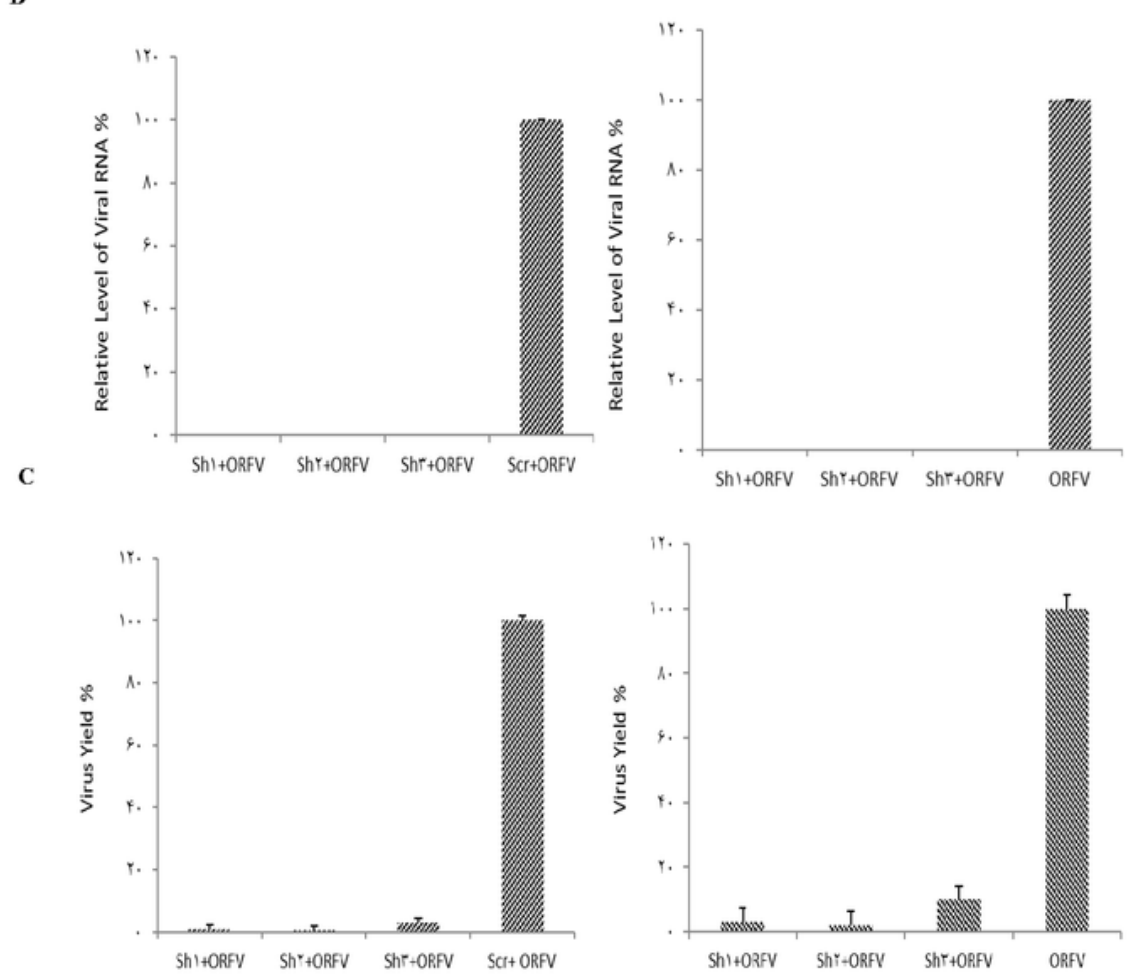

Fig. 1

\section{Figure 1}

A. Reduction of ORFV replication by shRNAs. The MDBK cells coinfected with lentivectors expressing shRNA and ORF virus. 72 hours after infection, the CPE of ORFV was visualized with a light microscope. ShRNA 1,2 and 3 reduced cytopathic effects of ORFV and MDBK cells had a relatively normal morphology while in the wells infected by ORFV the cell morphology was changed. B. Reducing ORFV by recombinant shRNA lentivirus: Extracted total RNA was used for real-time RT-PCR analysis. All the values 
were displayed in percentages of controls (cells infected with ORFV and those infected with Scrambled vector). The shRNAs 1, 2 and 3 noticeably decreased the expression of viral RNA compared to cells infected with ORFV and scrambled vector (Left chart) and compared to cells infected with ORFV (Right chart). C. TCID50 assay: The titration of virus was detected at $48 \mathrm{~h}$ pi. The viral yields were measured by the ratio of TCID50 of expressed shRNAs to that of controls (cells infected with ORFV and those infected by Scrambled vector). Error bars show standard deviation of three independent experiments. The shRNAs 1, 2 and 3 reduced virus yield compared to cells infected with ORFV (Left chart) and compared to cells infected with ORFV and scrambled vector (Right chart).
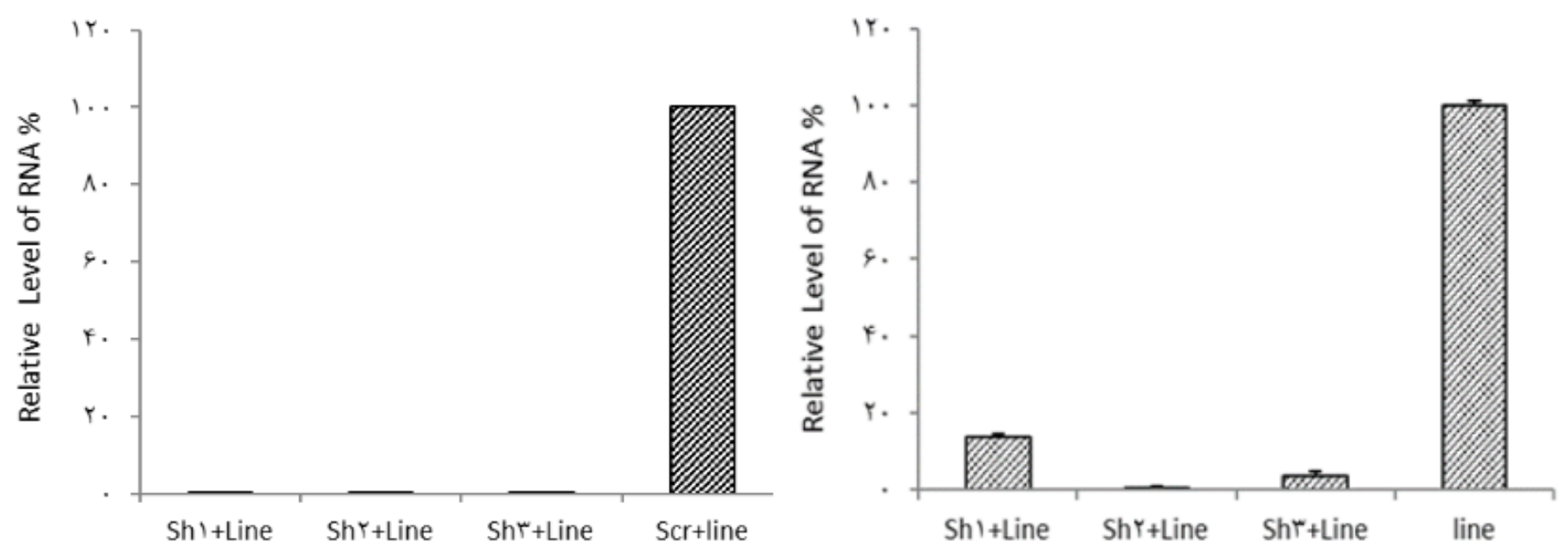

Figure 2

The inhibition of ORFV DnaQ_like expression by lentiviral expressing shRNA in MDBK cells. The total RNA was extracted and real-time RT-PCR was used for the determination of relative RNA. All the values were displayed in percentages of controls (cells infected with ORFV and those infected by Scrambled vector). The shRNAs 1, 2 and 3 markedly reduced expression selected gene compared to the control groups compared to cells without any infection (Left chart) and compared to cells infected by scrambled vector (Right chart).

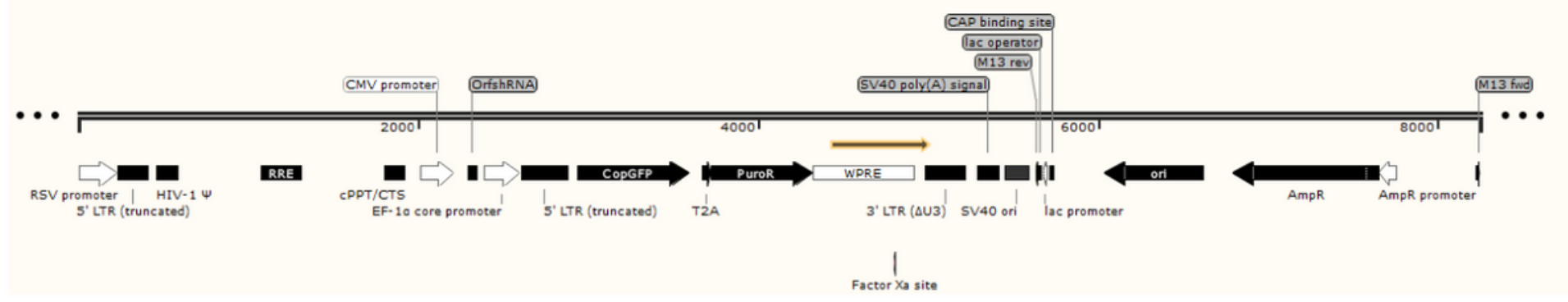

\section{Figure 3}


A
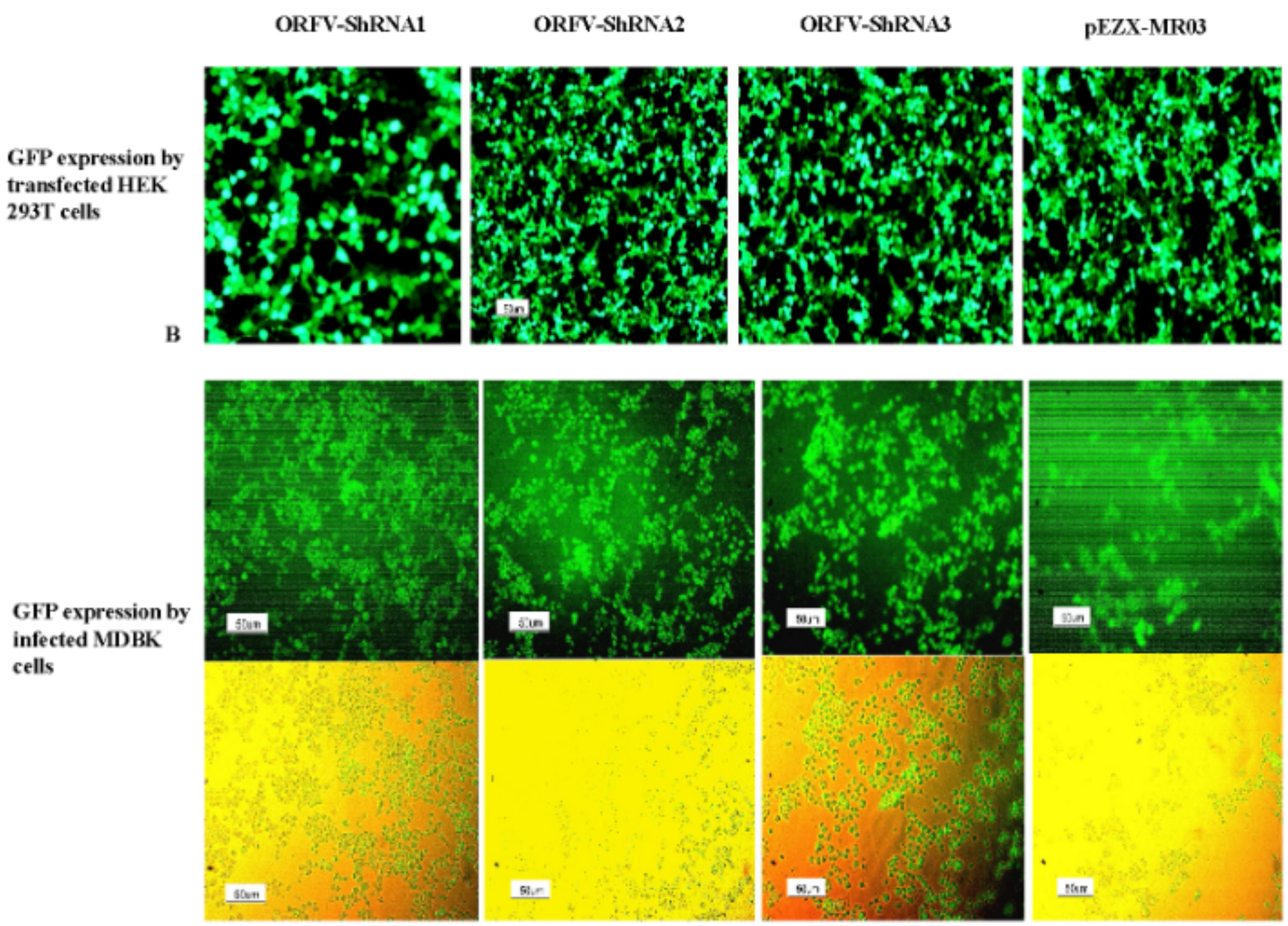

Figure 4

A. GFP expression after Co-transfection of HEK293T cells: GFP expression by transfected cells with psPAX and pMD2.G as packaging vectors and pCDH carrying ORFV-ShRNA1, pCDH carrying ORFVShRNA2, pCDH carrying ORFV-ShRNA3 and pEZX-MR03 (as a mock), respectively. B. GFP expression after infection MDBK cells with lentivectors: GFP expression by infected MDBK cells with lentivector expressing ORFV-ShRNA1, ORFV-ShRNA2, ORFV-ShRNA 3 and scrambled lentivector respectively and the same pictures with the light microscope. 